\title{
On the foundations of quantum physics
}

\author{
E. A. Solov'ev \\ Bogoliubov Laboratory of Theoretical Physics, Joint Institute for Nuclear Research, \\ 141980 Dubna, Moscow region, Russia \\ E-mail: esolovev@theor.jinr.ru
}

\begin{abstract}
Some aspects of the interpretation of quantum theory are discussed. It is emphasized that quantum theory is formulated in a Cartesian coordinate system; in other coordinates the result obtained with the help of the Hamiltonian formalism and commutator relations between 'canonically conjugated' coordinate and momentum operators leads to a wrong version of quantum mechanics. In this connection the Feynman integral formalism is also discussed. In this formalism the measure is not well-defined and there is no idea how to distinguish between the true version of quantum mechanics and an incorrect one; it is rather a mnemonic rule to generate perturbation series from an undefined zero order term. The origin of time is analyzed in detail by the example of atomic collisions. It is shown that the time-dependent Schrödinger equation for the closed three-body (two nuclei + electron) system has no physical meaning since in the high impact energy limit it transforms into an equation with two independent time-like variables; the time appears in the stationary Schrödinger equation as a result of extraction of a classical subsystem (two nuclei) from a closed three-body system. Following the Einstein-Rosen-Podolsky experiment and Bell's inequality the wave function is interpreted as an actual field of information in the elementary form. The relation between physics and mathematics is also discussed.
\end{abstract}

Eine neue wissenschaftliche Wahrheit pflegt sich nicht in der Weise durchzusetzen, dass ihre Gegner überzeugt werden und sich als belehrt erklären, sondern dadurch, dass die Gegner allmählich aussterben und dass die heranwachsende Generation von vornherein mit der Wahrheit vertraut gemacht ist.(In English: A new scientific truth does not triumph by convincing its opponents and making them see the light, but rather because its opponents eventually die, and a new generation grows up that is familiar with it.) 


\section{Introduction}

This paper is an extended version of my talk given at the International Conference "Symmetries in Physics" dedicated to the 90th anniversary of Ya.A. Smorodinsky (March 27 - 29 2008, Dubna, Russia) [1], where some general aspects in the interpretation of quantum theory are reviewed. This topic has an eighty-year history and many versions have been proposed, beginning with the Copenhagen interpretation. Here three new points are presented:

- Quantum theory is much less symmetric than classical mechanics. In contrast to classical mechanics, quantum theory is not covariant with respect to canonical transformations. Moreover, an analogue of a canonical transformation cannot be introduced into quantum mechanics.

- The time-dependent Schrödinger equation for closed systems is an artifact and has no fundamental meaning; the time appears in the stationary Schrödinger equation as a result of extraction of a classical subsystem from a total system.

- Quantum theory, in its origin, deals with a new object - "field of information" which is additional to the objects "material point" introduced in classical mechanics and "electromagnetic field" introduced in classical electrodynamics.

All these statements are not a personal opinion - they are proved at the standard physical level.

Within the last point the role of the concept of 'measurement' and its connection with the way we take a decision (or how our brain acts) are analyzed. In the conclusion, the relation between physics and mathematics is also discussed.

Here the philosophical and popular science papers related to this topic are not discussed since they are not based on the laws of nature but on the metaphysical assumptions which are beyond the natural-science standards. A very nice description of such approach one may find in Feynman's book [2], Chapter 'Cargo Cult Science'.

\section{Symmetry in quantum and classical physics}

The Schrödinger equation is given in the Cartesian coordinate system $\{x, y, z\}$

$$
\left[-\frac{\hbar^{2}}{2 m}\left(\frac{\partial^{2}}{\partial x^{2}}+\frac{\partial^{2}}{\partial y^{2}}+\frac{\partial^{2}}{\partial z^{2}}\right)+V(\boldsymbol{r})\right] \Psi_{n}(\boldsymbol{r})=E_{n} \Psi_{n}(\boldsymbol{r})
$$

The exclusive role of Cartesian coordinates is quite well-known (see, e.g., [3]). Equation (2.1) is a law of nature and it cannot be derived. In all other coordinates (or representations) the result is obtained by a mathematical transformation of equation (2.1) from the Cartesian coordinates to desirable coordinates (or representation) which, of course, does not change the physical (observable) results. An alternative way to introduce the Schrödinger equation in arbitrary coordinates based on 'canonical' transformations has serious problems. Generally, the momentum operator $\hat{p}$, defined in 
the $q$-representation by the commutator relation $q \hat{p}-\hat{p} q=i \hbar$, has the form

$$
\hat{p}=-i \hbar \frac{\partial}{\partial q}+f(q) \text {. }
$$

The function $f(q)$ is determined by Hermiticity condition in a given coordinate system

$$
\int \psi_{1}^{*}(q) \hat{p} \psi_{1}(q) J(q) d q=\int\left[\hat{p} \psi_{1}(q)\right]^{*} \psi_{1}(q) J(q) d q
$$

from which

$$
\hat{p}=-i \hbar \frac{1}{\sqrt{J(q)}} \frac{\partial}{\partial q} \sqrt{J(q)},
$$

where $J(q)$ is the Jacobian. In the spherical coordinates $\{r, \vartheta, \varphi\}$ the 'canonically conjugated' Hermitian components of the momentum operators are

$$
\hat{p}_{r}=-i \hbar \frac{1}{r} \frac{\partial}{\partial r} r, \quad \hat{p}_{\vartheta}=-i \hbar \frac{1}{\sqrt{\sin \vartheta}} \frac{\partial}{\partial \vartheta} \sqrt{\sin \vartheta}, \quad \hat{p}_{\varphi}=-i \hbar \frac{\partial}{\partial \varphi} .
$$

However, if we substitute $\hat{p}_{r}, \hat{p}_{\vartheta}$ and $\hat{p}_{\varphi}$ in the operator of kinetic energy, an incorrect result is obtained:

$$
\hat{T}=\frac{1}{2 m}\left[{\hat{p_{r}}}^{2}+\frac{1}{r^{2}}\left({\hat{p_{\vartheta}}}^{2}+\frac{{\hat{p_{\varphi}}}^{2}}{\sin ^{2} \vartheta}\right)\right]=-\frac{\hbar^{2}}{2 m}\left(\Delta+\frac{\cos ^{2} \vartheta-2}{4 r^{2} \sin ^{2} \vartheta}\right),
$$

since a term additional to the Laplacian appears, i.e., in the simplest case of transformation from the Cartesian to spherical coordinates the Hamiltonian formalism (which is mostly coordinate-momentum variables plus covariance of the theory with respect to the canonical transformation) does not work. Thus, the momentum operator, as well as the Hamiltonian formalism, is not well defined.

The incompatibility of the canonical transformations with quantum theory becomes obvious if we consider the Bohr-Sommerfeld quantization rule

$$
\int_{q_{1}}^{q_{2}} p(q) d q=\pi \hbar\left(n+\alpha_{1}+\alpha_{2}\right)
$$

where $q_{i}$ is a turning point (caustic, which is a non-local characteristic of trajectory) and $\alpha_{i}$ is a quantum phase shift in it. Here the left-hand side is invariant with respect to the canonical transformation whereas the right-hand side changes since caustics can disappear under canonical transformation, for instance, after transformation to actionangle variables. In [4], the action-angle variables are used to obtain the semiclassical energy spectrum for the quadratic Zeeman effect. However, in these variables the information about caustics is absent, and the quantization condition (3.8) in [4] erroneously employs an integer quantum number instead of semi-integer which leads to a wrong energy spectrum. The correct semiclassical energy spectrum is obtained in configuration space [5]. Thus, the canonical transformations are not compatible even with the Bohr-Sommerfeld quantization condition.

Many textbooks ignore these facts treating the commutator relation between coordinate and generalized momentum operators as a background of quantum mechanics (see, e.g., [6]) or describing the states by the Feynman integral (see, e.g., [7]). In particular, the Feynman integral cannot be rigorously determined since the measure 
of this integral does not exist as a mathematical quantity. From the physical point of view the measure cannot be introduced since in this approach there is no idea how to distinguish the true version of quantum mechanics from an incorrect one; it is rather a mnemonic rule to generate perturbation series from an undefined zero order term. All these treatments provoke a wrong opinion that quantum theory has the same level of symmetry as classical mechanics.

\section{Stationary and time-dependent Schrödinger equation}

In 1926 Schrödinger 8 formulated quantum theory as an eigenvalue problem for the Hamiltonian operator

$$
\hat{H} \Psi_{n}=E_{n} \Psi_{n} .
$$

The validity of this representation was confirmed, e.g., by comparison of the obtained energy spectrum of the hydrogen atom with experimental data. In [9], Schrödinger introduced the time-dependent equation

$$
\hat{H} \Psi=i \hbar \frac{\partial}{\partial t} \Psi
$$

However, the Hamiltonian operator does not contain the time, and equation (3.2) looks like an artificial constructiont Besides, from the general point of view, time is not observable in quantum theory - there is no time-like Hermitian operator. Some aspects of this problem were discussed, e.g., in [10] and [11]. Here the emergence of time in atomic collision theory is analyzed in detail.

The inelastic transitions of an electron in an atomic collision are described in the c.m. frame of reference by the Schrödinger equation

$$
\hat{\mathcal{H}} \Psi(\boldsymbol{R}, \boldsymbol{r})=\mathcal{E} \Psi(\boldsymbol{R}, \boldsymbol{r}),
$$

where

$$
\hat{\mathcal{H}}=-\frac{\hbar^{2}}{2 M} \Delta_{\boldsymbol{R}}+\frac{Z_{1} Z_{2}}{R}+\hat{H}_{e l .}(\boldsymbol{R})
$$

is the three-body Hamiltonian operator, $Z_{1}, Z_{2}$ are the charges and $M$ is the reduced mass of the nuclei, $\boldsymbol{R}$ is the internuclear radius-vector, $\boldsymbol{r}$ is the radius-vector of electron referred to c.m. of nuclei, $\hat{H}_{e l}(\boldsymbol{R})$ is the electronic part of the total Hamiltonian. In the adiabatic representation the wave function $\Psi(\boldsymbol{R}, \boldsymbol{r})$ has the form $\xi$ :

$$
\Psi(\boldsymbol{R}, \boldsymbol{r})=\sum_{n, L} \frac{F_{n}^{L}(R)}{R} Y_{L 0}(\theta, \varphi) \phi_{n}(\boldsymbol{r} ; \boldsymbol{R}),
$$

where $Y_{L M}(\theta, \varphi)$ are spherical functions (angular part of the nuclear wave function), $\phi_{n}(\boldsymbol{r} ; \boldsymbol{R})$ are the adiabatic wave functions, and $E_{n}(R)$ are the adiabatic energies -

$$
\hat{H}_{e l .}(\boldsymbol{R}) \phi_{n}(\boldsymbol{r} ; \boldsymbol{R})=E_{n}(R) \phi_{n}(\boldsymbol{r} ; \boldsymbol{R})
$$

$\ddagger$ For instance, initially Schrödinger introduced an equation of second order in the time derivative.

$\S$ It is assumed that the impact velocity is directed along the $z$-axis. 
which depend on $\boldsymbol{R}$ as an external parameter in the Hamiltonian $\hat{H}_{e l}(\boldsymbol{R})$. After the substitution of (3.5) into (3.3), the Schrödinger equation takes the form of a system of equations for the radial nuclear wave functions

$\left[-\frac{\hbar^{2}}{2 M} \frac{d^{2}}{d R^{2}}+\frac{\mathcal{L}^{2}}{2 M R^{2}}+\frac{Z_{1} Z_{2}}{R}+E_{n}(R)-\mathcal{E}\right] F_{n}^{L}(R)=\sum_{n^{\prime} L^{\prime}} \hat{W}_{n n^{\prime}}^{L L^{\prime}}(R) F_{n^{\prime}}^{L^{\prime}}(R)$,

where $\mathcal{L}^{2}=\hbar^{2} L(L+1)$ is the squared nuclear angular momentum. The operator of the non-adiabatic coupling $\hat{W}_{n n^{\prime}}^{L L^{\prime}}$ is equal to

$$
\begin{aligned}
& \hat{W}_{n n^{\prime}}^{L L^{\prime}}=\frac{\hbar^{2}}{2 M}\left[\left(2\left\langle n\left|\frac{\partial}{\partial R}\right| n^{\prime}\right\rangle \frac{\partial}{\partial R}+\left\langle n\left|\frac{\partial^{2}}{\partial R^{2}}\right| n^{\prime}\right\rangle+\frac{1}{R^{2}}\left\langle n\left|\frac{\partial^{2}}{\partial \theta^{2}}\right| n^{\prime}\right\rangle\right) \delta_{L L^{\prime}}+\right. \\
& \left.+\frac{2 i}{\sqrt{2 L+1} R^{2}}\left\langle n\left|\frac{\partial}{\partial \theta}\right| n^{\prime}\right\rangle\left(\frac{L^{2}}{\sqrt{2 L-1}} \delta_{L, L^{\prime}+1}+\frac{(L+1)^{2}}{\sqrt{2 L+3}} \delta_{L, L^{\prime}-1}\right)\right]
\end{aligned}
$$

where $\left\langle n|\hat{A}| n^{\prime}\right\rangle=\int \phi_{n}(\boldsymbol{r} ; \boldsymbol{R}) \hat{A} \phi_{n^{\prime}}(\boldsymbol{r} ; \boldsymbol{R}) d \boldsymbol{r}$ is the matrix element of the operator $\hat{A}$ between $|n\rangle$ and $\left|n^{\prime}\right\rangle$ adiabatic electronic states.

In the semiclassical limit $(\hbar \rightarrow 0, L \rightarrow \infty)$ the solution has an essential singularity which can be extracted explicitly:

$$
F_{n}^{L}(R)=\tilde{F}_{n}^{L}(R) e^{-\frac{i}{\hbar} S_{n}(R)}
$$

where $S_{n}(R)=\int^{R} \mathcal{P}_{n}\left(R^{\prime}\right) d R^{\prime}$ is the classical action and

$$
\mathcal{P}_{n}(R)=\sqrt{2 M\left[\mathcal{E}-\frac{\mathcal{L}^{2}}{2 M R^{2}}-\frac{Z_{1} Z_{2}}{R}-E_{n}(R)\right]}
$$

is the radial momentum of the nuclei in the $n$-channel. In terms of $\tilde{F}_{n}^{L}(R)$ the Schrödinger equation (3.3) has the form $\left(\Delta S_{n n^{\prime}}(R)=S_{n}(R)-S_{n^{\prime}}(R)\right)$

$$
i \hbar \frac{\mathcal{P}_{n}(R)}{M} \frac{d \tilde{F}_{n}^{L}(R)}{d R}-\frac{\hbar^{2}}{2 M} \frac{d^{2} \tilde{F}_{n}^{L}(R)}{d R^{2}}=\sum_{n^{\prime} L^{\prime}} e^{\frac{i}{\hbar} \Delta S_{n n^{\prime}}(R)} \hat{W}_{n n^{\prime}}^{L L^{\prime}}(R) \tilde{F}_{n^{\prime}}^{L^{\prime}}(R),
$$

Since $\tilde{F}_{n}^{L}(R)$ is a smooth function with respect to $\hbar \rightarrow 0$, we can neglect the second term in the left-hand side of this equation. In the right-hand side the dominant contribution is obtained through differentiation of exponent $e^{-i S_{n^{\prime}}(R) / \hbar}$ over $R$ (first term in (3.8)) and if we neglect the difference between $L$ and $L \pm 1$ in the second line of expression (3.8). In this limit the nuclear angular momentum is conserved and equation (3.11) takes the form

$i \hbar \frac{\mathcal{P}_{n}(R)}{M} \frac{d \tilde{F}_{n}^{L}(R)}{d R}=i \hbar \sum_{n^{\prime}}\left\langle n\left|\frac{\mathcal{P}_{n^{\prime}}(R)}{M} \frac{\partial}{\partial R}+\frac{\mathcal{L}}{M R^{2}} \frac{\partial}{\partial \theta}\right| n^{\prime}\right\rangle e^{\frac{i}{\hbar} \Delta S_{n n^{\prime}}(R)} \tilde{F}_{n^{\prime}}^{L}(R)$.

If $\mathcal{E} \gg E_{n}(R)$, the classical approximation for nuclear motion can be employed. Treating $E_{n}(R)$ as a small quantity, the momentum $\mathcal{P}_{n}(R)$ can be approximated [10]:

$$
\mathcal{P}_{n}(R) \approx M V(R)
$$

where $V(R)$ is the radial nuclear velocity without $E_{n}(R)$. Then the factor $M d R / \mathcal{P}_{n}(R) \approx d R / V(R)=d t$ has the meaning of the differential of time, the exponent 
$\Delta S_{n n^{\prime}}(R) \approx \int^{t} \Delta E_{n n^{\prime}}\left(R\left(t^{\prime}\right)\right) d t^{\prime}\left(\Delta E_{n n^{\prime}}(t)=E_{n}(R(t))-E_{n^{\prime}}(R(t))\right)$ and the Schrödinger equation takes the well-known time-dependent form

$$
\begin{aligned}
& i \hbar \frac{d}{d t} \tilde{F}_{n}^{L}(t)=i \hbar \sum_{n^{\prime}}\left\langle n\left|\frac{d R(t)}{d t} \frac{\partial}{\partial R}+\frac{d \theta(t)}{d t} \frac{\partial}{\partial \theta}\right| n^{\prime}\right\rangle \tilde{F}_{n^{\prime}}^{L}(t) e^{-\frac{i}{\hbar} \int^{t} \Delta E_{n n^{\prime}}\left(t^{\prime}\right) d t^{\prime}}= \\
& =i \hbar \sum_{n^{\prime}}\left\langle n\left|\frac{d}{d t}\right| n^{\prime}\right\rangle \tilde{F}_{n^{\prime}}^{L} e^{-\frac{i}{\hbar} \int^{t} \Delta E_{n n^{\prime}}\left(t^{\prime}\right) d t^{\prime}}
\end{aligned}
$$

where the classical definition of angular momentum $\mathcal{L}=M R^{2} d \theta(t) / d t$ was used. In [12], equation (3.13) was obtained from the time-dependent Schrödinger equation

$$
\hat{H}_{e l .}(\boldsymbol{R}(t)) \psi(\boldsymbol{r}, t)=i \hbar \frac{\partial}{\partial t} \psi(\boldsymbol{r}, t)
$$

after substitution wave function in the form

$$
\psi(\boldsymbol{r}, t)=\sum_{n} \tilde{F}_{n}^{L}(t) e^{-\frac{i}{\hbar} \int^{t} E_{n}\left(t^{\prime}\right) d t^{\prime}} \phi_{n}(\boldsymbol{r} ; \boldsymbol{R}(t)) .
$$

Thus, the time-dependent Schrödinger equation (3.14) is derived straightforwardly from the stationary Schrödinger equation (3.3). From the above considerations it is clear that 'time' can be introduced in the high impact energy limit only when we can neglect the dependence of $\mathcal{P}_{n}(R)$ on $n$. At low impact energy, the solution of the Schrödinger equation (3.7) is the superposition of nuclear motions in different $n$-channels having significantly different momenta $\mathcal{P}_{n}(R)$ and a unique time does not exist.

Now let us consider the time-dependent equation

$$
\hat{\mathcal{H}} \Psi(\boldsymbol{R}, \boldsymbol{r}, t)=i \hbar \frac{\partial}{\partial t} \Psi(\boldsymbol{R}, \boldsymbol{r}, t)
$$

instead of the stationary Schrödinger equation (3.3). If we apply the same approximation as above, we obtain an analogue of equation (3.13) which has two independent timelike variables: one existing in the initial form of equation (3.16) and the other coming from the second derivative with respect to $R$, i.e. this equation is meaningless. Just the extraction of the classical subsystem from the total system in the stationary Schrödinger equation leads to the replacement of the coordinates of the classical subsystem by the time variable. It is the sole reason for emergence of the actual time in the Hamiltonian operator. This analysis plays the role of experimentum crucis in the clarification of the place of the time in quantum theory. In the simpler case of the two-body problem the conflict between stationary (3.1) and time-dependent (3.2) equations is obscure since inelastic channels do not exist and we cannot extract a classical subsystem.

In the scattering theory (two-body problem) the time-dependent Schrödinger equation is employed to avoid the normalization problem in the continuum (see, e.g., [13]) - the stationary problem is out of the Hilbert space and we have no well-defined physical stationary states. It is a very serious defect of the theory. Below the boundary of the continuum, the aim of the theory is the search for stable (or stationary) states which exist at discrete energy values only. Above the boundary of continuum the problem is completely different. The concept 'stable' or 'unstable' does not exist here. The solution of the stationary Schrödinger equation cannot be normalized to unit. In 
classical approach the particle comes from infinity and then goes to infinity as $t \rightarrow \infty$; probability to find it at any finite distance is equal to zero and to find it at infinity is equal to unit. Some tricky way to resolve this problem is to introduce the wave packet formalism. However, as was shown above, the 'time' variable in this formalism has no direct physical meaning; the wave packet construction plays rather a psychological role. At the same time, all physical results in the scattering theory are derived from the stationary Schrödinger equation.

Generally, the presence of the time in the time-dependent Schrödinger equation means that some subsystem is under our non-stop control since the time is part of the frame of reference which is a tool for measurement. The connection between two frames of reference (Galileo or Lorenz transformation) is also a classical concept which is beyond quantum theory. Quantum theory describes a new object - the field of information (see the next section) which has no relation to the time for closed systems.

\section{The information field and measurement}

In classical mechanics and classical electrodynamics the concepts 'material points' and 'electro-magnetic waves' were introduced. In this sense we can consider quantum theory as a theory of an 'information field' (see, e.g., [1]) which is a wave function $\Psi(x, y, z)$. At first sight this statement looks non-persuasive, but this case is similar to the situation with the electro-magnetic field - nobody suspected its existence before classical electrodynamics. The interaction between 'material points' and 'information field' is the act of measurement. Thus, 'information field' is an additional object to 'material points' and 'electro-magnetic waves' but without mass and energy. This statement is in contradiction with wave-particle duality. In the present interpretation we have material points characterized by the coordinates $x, y, z$, and the 'information field' $\Psi(x, y, z)$ which is subject to the wave-type Schrödinger equation. Thus, there is no duality because of two different objects - the material points and the information field. Experimentum crucis proving the existence of the information field, is based on the Einstein-Podolsky-Rosen phenomenon [14] and Bell's inequality [15] which allows one to distinguish whether the quantum approach is complete or not. One of the realizations of this experiment is the following. After a radiative decay of a hydrogen atom from a metastable $2 S$-state two photons are emitted having spins with opposite directions

$$
s_{1}=-s_{2},
$$

since the initial $(2 S)$ and final $(1 S)$ states of hydrogen have angular momentum equal to zero. However, the orientation of each spin is uncertain in the same manner as the position of a particle in a potential well. If the measurement of the direction of the spin of the first photon is performed, the second photon takes the opposite direction of spin, according to (4.1), independently of the distance between photons. The fixation of the 
spin orientation of the second photon happens immediately $\lfloor$ and it is an actual changing of its state which is confirmed by experiment [16], where it was found that the speed of quantum information is at least $10^{4}$ greater than the speed of light $c$. Measurement gives orientation of spin, according to this field of information. Intuitively, it is clear what the concept 'measurement' means but it cannot be formalized, i.e., to be written in a mathematical form. The nontrivial role of measurement was most clearly realized in quantum physics.

The fresh demonstration of the existence of a common information field is the effect of long-range near-side angular correlations in proton-proton collisions at the LHC in CERN [17].

The interpretation of the wave function as a common field of information makes the well-known facts such as a collective behavior of bees and ants, bird navigation, extremely fast baby's mental development, déjà vu phenomenon etc. more transparent. Perhaps such a object (common field of information) could clarify how our brain acts. Here the statement made by Svyatoslav Medvedev (Director of the Institute of Human Brain, Russian Academy of Sciences) in the TV program dedicated to the memory of the Russian psychiatrist Vladimir Bekhterev can be cited: " ... our brain is the interface between the material and the spiritual world ...". In this context, brain activity can be interpreted as follows: consciousness prepares the incoming information into an appropriate form to send it to subconsciousness (common information field level). What happens in subconsciousness is hidden from our mind, but the final decision suddenly appears (sometimes after a long time - depending on the level of the problem). This event is similar to the spin measurement in the Einstein-Podolsky-Rosen experiment and can be considered as the most fundamental form of 'measurement'. Here our brain as an 'interface' plays the role of a tool of measurement.

Generally, we do not know so much about consciousness even with respect to Socrates. Modern set of tools in this area such as measurement of temperature, blood pressure, electroencephalography etc. are taken from inorganic natural sciences. All of them have no direct relation to conscious activity and memory; it is the same as during the Hellenistic period to try to investigate consciousness by means of a ruler and balance. That is why at the end of her life Natalia Bekhtereva (Director of the Institute of Human Brain 1990-2008) said $\mathbf{q}$ : "Doesn't exist yet not only theory, but even a reasonable hypothesis how the brain works". In addition, a scientific approach is based on the assumption that physical events are reproduced at any place and any moment under the same external conditions. In this sense consciousness as well as memory cannot be a subject of science since it is personal and cannot be repeated. Besides, we have no idea where they are located. Moreover, it seems that space and matter are irrelevant here. The only vague way to contemplate the consciousness is perhaps through analysis of our dreams which are out of our autocontrol and instincts.

\| This does not contradict the relativistic restriction $v \leq c$ because the carrier of information is not material, i.e., it has no relation to the transfer of mass or energy.

ฯ Interview in the Russian newspaper "Rossiyskaya gazeta" from June, 26th 2003 
That is why often we get the decision during the dream.

\section{Physics and mathematics}

Physics as science was founded by Galileo Galilei. He discovered the fundamental role of experiments in inorganic nature - physical events are reproduced at any place and any moment under the same external conditions, i.e. they obey the laws of nature which take the form of mathematical equations introduced by Isaac Newton. Thus, physical theories are based on mathematics which is a logical scheme related rather to our mentality than to nature. It is a miracle but mathematics works! Mathematics is based on a few axioms such as $a+b=b+a, c(a+b)=c a+c b,(a+b)+c=a+(b+c)$ and so on. In principle, mathematics does not produce new information; its aim is the transformation, according to these axioms, of the initial expression to a form more transparent for our consciousness, e.g., by solution of a differential equation. Thus, mathematics plays a subordinate, auxiliary role in physics. Investigations based only on the abstract mathematical background lead, sometimes, to artefacts.

The first example is the case of irregular motion in classical mechanics. In spite of a huge number of papers devoted to this problem there is no important achievement for real physical systems. Irregular motion is mostly studied in 2D billiard models and 1.5D models which are in fact non-Hamiltonian systems. Interest in the irregular motion is explained by an attempt to understand the origin of the arrow of time as provided by the Second Law of Thermodynamics which says that in an isolated system entropy tends to increase with time. However, there is a fundamental contradiction between such evolution and classical mechanics - the first is $100 \%$ irreversible in time whereas classical mechanics is $100 \%$ reversible. Thus, classical mechanics rather deals with pseudo-chaotic evolution which is due to the fundamental difference between rational and irrational numbers in mathematics which does not exist in nature. Generally speaking, the axioms of mathematics provide always reversible time behaviour. We can obtain irreversible evolution only if we introduce a stochastic concept such as 'probability' which is beyond standard mathematics.

The next example is Gutzwiller's 'theory'. In this approach, the contribution of the unstable periodic orbit to the trace of the Green function is determined by the formula [18]

$$
g(E) \sim-\frac{i T(E)}{2 \hbar} \sum_{n=1}^{\infty} \frac{\exp \{i n[S(E) / \hbar-\lambda \pi / 2]\}}{\sinh [n w(E) / 2]}
$$

where $S(E), w(E), T(E)$ and $\lambda$ are the action, the instability exponent, the period and the number of focal points during one period, respectively. After the expansion of the denominator, according to $[\sinh (x)]^{-1}=2 e^{-x} \sum_{k=0}^{\infty} e^{-2 k x}$, and summation of the geometric series over $n$ one can see that the response function (5.1) has poles at complex energies $E_{k s}$ whenever

$$
S\left(E_{k s}\right)=\hbar \lambda \pi / 2-i \hbar w\left(E_{k s}\right)\left(k+\frac{1}{2}\right)+2 s \pi \hbar, \quad k, s=0,1,2, \ldots
$$




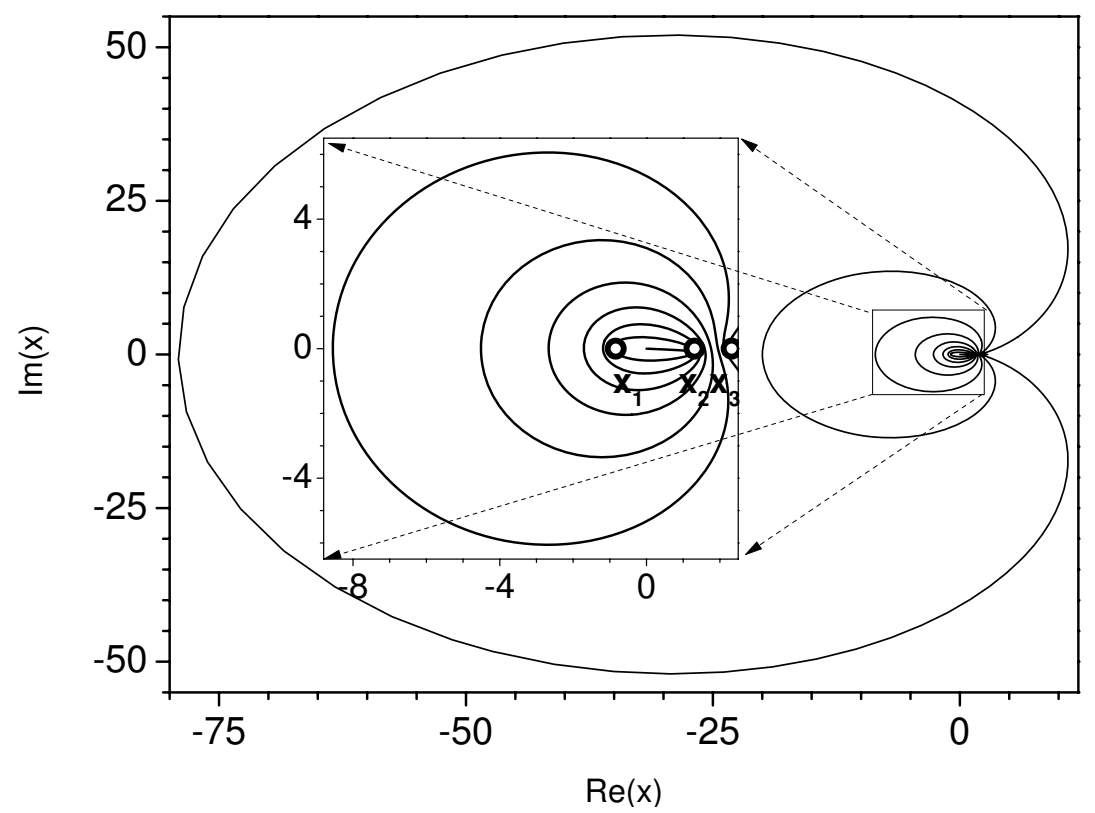

Figure 1. The trajectory in the complex x-plane of a classical particle with complex energy (5.4) at $g=2 / \sqrt{125}$. Open circles in the insertion show the turning points $x_{1}$, $x_{2}$ and $x_{3}$.

In fact, expression (5.1) has been introduced $a d$ hoc; it is based on local characteristics in the vicinity of the periodic orbit ignoring the asymptotic region which is responsible for the physical boundary condition. Thus, in this scheme it is impossible to distinguish whether the energy belongs to the discrete spectrum or the continuum. Besides, in nonseparable systems unstable periodic orbits with long periods lie everywhere dense in phase space (renormalization-group) and the response function (5.1) has a pathological structure like the Weierstrass function which is continuous everywhere but differentiable nowhere. The singularities predicted by expression (5.1) have no physical meaning in the case of the discrete spectrum as well, since the energies $E_{k s}$ are complex.

Recently, in [19] it has been argued that "quantum" effects can be explained in terms of classical trajectories. In particular, the tunneling effect for a potential

$$
V(x)=x^{2} / 2-g x^{3}
$$

has been discussed. In this case, the lower state is a quasi-stationary state whose lifetime $\tau$, defined by the population of the bound state $P(t)=e^{-t / \tau}$, can be approximated for small $g$ using WKB theory by the expression 20]

$$
\tau=\frac{1}{2} g \sqrt{\pi} \exp \left(2 / 15 g^{2}\right)
$$

In Fig.8 of the paper [19], a complex classical trajectory with complex energy (5.4) at $g=2 / \sqrt{125}$ is represented (see also insertion in Fig.1 of this paper $\uplus$ ) with comment

"... initially, as the particle crosses the real axis to the right of the middle turning point,

+ Fig.1 and Tab.1 have been prepared by Alexander Gusev 
Table 1. The classical time $t_{c}$ (5.5) and the quantum lifetime $\tau$ (5.4) versus coupling constant $g$.

\begin{tabular}{|r|r|r|r|r|}
\hline$g$ & 0.12522 & 0.14311 & 0.16099 & 0.17888 \\
\hline$t_{c}$ & 15009 & 1385 & 220 & 49 \\
$\tau$ & 547.3 & 85.2 & 24.4 & 10.2 \\
\hline
\end{tabular}

its trajectory is concave leftward, but as time passes, the trajectory becomes concave rightward. It is clear that by the fifth orbit the right turning point has gained control, and we can declare that the classical particle has now 'tunneled' out and escaped from the parabolic confining potential. The time at which this classical changeover occurs is approximately at $t=40$. This is in good agreement with the lifetime of the quantum state in (8), whose numerical value is about 20."

However, if the time increases further, one can see from Fig.1 that the trajectory does not escape toward the right side but continues to rotate mostly on the opposite side. On the other hand, we can compare the quantum lifetime $\tau$ (5.4), and the classical time to reach the right turning point $t_{c}$ -

$$
\operatorname{Re} x\left(t_{c}\right)=\operatorname{Re} x_{3}
$$

- proposed in [19] as a classical analogue of $\tau$. It is seen from Table 1 that there is nothing in common between these two quantities in spite of the "good agreement" declared in [19]. Thus, the effect discussed is an artificial result which has no relation to quantum theory.

\section{Concluding remarks}

Any theory based on mathematics has internal conflicts. Probably, only classical mechanics has no evident contradictions. However, classical electrodynamics is not selfconsistent in its background - an accurate dynamic equation describing the common evolution of charged particles and the electro-magnetic field does not exist since the concept 'material point' leads to the divergence of the energy of its electro-magnetic field, and, on the other hand, the concept 'solid state' is in conflict with relativistic principles. Here we need a more sophisticated concept than 'material point' and 'solid state' which is maybe beyond the standard mathematical language. In quantum mechanics, we cannot clearly formulate a physical stationary state in the continuum (see Sec.3).

A scientific approach is quite restricted since our vocabulary is not certain and complete in principle. For instance, it admit such paradoxes as "Can God create a stone so heavy that he cannot lift it?". Another example which demonstrates the incompleteness of vocabulary is the fact that physics cannot be represented in Eskimo language where they have dozen of words for different types of snow (snow which fell down yesterday, snow on which a dog-sledge passed, etc.); however, there is no word 
'snow' which is too abstract for them. But what is the level of our mentality (language $\rightleftharpoons$ mentality)? However it is the sole tool for communication. In mathematics, the trace of this incompleteness is Gödel's theorem [21] which states that there are true propositions about the naturals that cannot be proved from the axioms.

\section{Acknowledgment}

I am grateful to Vladimir Belyaev, John Briggs, Tasko Grozdanov and participants of the Demkov, Ponomarev and BLTP seminars for fruitful discussions.

\section{References}

[1] Solov'ev E.A., Phys. At. Nuc. 72, 853 (2009)

[2] Feynman R.P., Surely You're Joking, Mr. Feynman!, W. W. Norton \& Co (1985)

[3] Fock V.A., Nachala kvantovoj mehaniki, LGU Leningrad (1932), p.43 (in Russian); Sommerfeld A., Atombau und Spectrallinien, II Band, Friedr.Vieweg \& Sohn. (1951), §6 (in German)

[4] Richards D., J. Phys. B 16, 749 (1983)

[5] Solov'ev E.A., Sov. Phys. - JETP 56, 1017 (1982)

[6] Dirac P.A.M., The Principles of Quantum Mechanics, Yeshiva University New York (1964)

[7] Feynman R.P., Hibbs A.R., Quantum Mechanics and Path Integrals, McGraw-Hill Book Company New York (1965)

[8] Schrödinger E., Ann. Phys. 79, 361 (1926)

[9] Schrödinger E., Ann. Phys. 81, 109 (1926)

[10] Solov'ev E.A., The Physics of Electronic and Atomic Collisions, XIX ICPEAC, p.471 (1995)

[11] Briggs J.S., Boonchui S., Khemmani S., J. Phys. A 40, 1289 (2000)

[12] Born M., Fock V., Zs. Phys. 51, 165 (1928)

[13] Newton R.G., Scattering Theory of Waves and Particles, McGraw-Hill Book Company New York (1982)

[14] Einstein A., Podolsky B., Rosen N., Phys. Rev. 47, 777 (1935)

[15] Bell J.S., Physics 1, 195 (1964)

[16] Salart D., Baas A., Branciard C., Gisin N., Zbinden H., Nature 454 861, (2008)

[17] CMS Collaboration, at arXiv:1009.4122 (2010)

[18] Gutzwiller M.C., J. Math. Phys. 12343 (1971)

[19] Bender C.M., Brody D.C. and Hook D.W., J. Phys. A 41352003 (2008)

[20] Bender C.M., Yaris R., Bendler J., Lovett R. and Fedders P.A., Phys. Rev. A 181816 (1978)

[21] Gödel K., Monatshefte für Mathematik und Physik 38, 173 (1931) 